BNL-108381-2015-JA

\title{
Characterising CCDs with cosmic rays
}

\section{Fisher-Levine ${ }^{1}$ and A. Nomerotski}

Brookhaven National Laboratory, Upton, NY 11973, U.S.A.

E-mail: mfisherlevine@bnl.gov

ABSTRACT: The properties of cosmic ray muons make them a useful probe for measuring the properties of thick, fully depleted CCD sensors. The known energy deposition per unit length allows measurement of the gain of the sensor's amplifiers, whilst the straightness of the tracks allows for a crude assessment of the static lateral electric fields at the sensor's edges. Furthermore, the small volume in which the muons deposit their energy allows measurement of the contribution to the PSF from the diffusion of charge as it drifts across the sensor. In this work we present a validation of the cosmic ray gain measurement technique by comparing with radioisotope gain measurments, and calculate the charge diffusion coefficient for prototype LSST sensors.

KEYWORDS: Photon detectors for UV, visible and IR photons (solid-state) (PIN diodes, APDs, Si-PMTs, G-APDs, CCDs, EBCCDs, EMCCDs etc); Instrument optimisation

\footnotetext{
${ }^{1}$ Corresponding author.
} 


\section{Contents}

1 Introduction to dark images 1

2 Gain measurement 2

2.1 Track selection 2

2.2 Gain measurement 2

3 PSF and charge diffusion 5

3.1 Track selection 5

3.2 PSF measurement 5

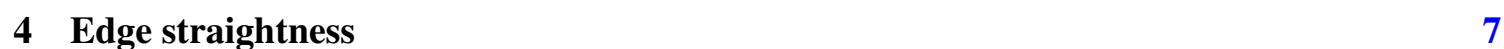

5 Summary $\quad 8$

\section{Introduction to dark images}

The abundance of cosmic ray muons at ground-level is a nuisance for ground-based astronomy; tracks left by these particles as they traverse sensors must be identified and removed before the images can be used. These tracks, however, also provide a useful tool for the characterisation of sensors, and some of the ways in they can be used are discussed here.

There are three basic types of events which are found in the dark exposures of thick, fullydepleted CCDs. When low-energy electrons are incident on a CCD, they are multiply scattered, and produce long ${ }^{1}$ winding tracks, which are commonly referred to as 'worms'. The source of these low-energy electrons is typically the Compton scattering of gamma rays from ${ }^{40} \mathrm{~K}$, and from isotopes from the $\mathrm{U}$ and Th decay chains [1]. Much smaller, diffusion-limited hits are also observed, which are referred to as 'spots', and are generally attributed to nuclear recoil events, very low-energy Compton scatters (short worms), or low energy x-rays. The third type of event found in these images, and the hardest to shield against, is the tracks of cosmic ray muons.

Cosmic rays are high-energy muons, typically created by the decay of pions in the upper atmosphere. They deposit energy in the sensor via the Coulomb interaction as they traverse the silicon lattice, with the average energy lost per unit length remaining approximately constant. The total energy loss is small relative to the high incoming energy, making them highly penetrating. This process is highly stochastic, with the energy deposit following the Landau distribution [2]. Due to the high momentum of these particles, the tracks they leave in the sensor are almost perfectly straight, and the energy is deposited in a very small volume, making these tracks appear sharp in the images, and it is these properties which make them useful tools for sensor characterisation.

\footnotetext{
${ }^{1}$ Long in this context means from several tens, up to one or two hundred pixels, in a $10 \mu \mathrm{m}$-pitch sensor.
} 


\section{Gain measurement}

The LSST focal plane will consist of $189100 \mu \mathrm{m}$ thick, $10 \mu \mathrm{m}$ pitch, science CCDs, each of which is read-out using 16 amplifiers [3], all of which will differ slightly in gain. In order to make precision photometric measurements, the relative gain of each of these amplifiers must be accurately measured.

The gain of astronomical CCDs is typically measured using either the photon transfer curve (PTC) method [4], or by using ${ }^{55} \mathrm{Fe}$ or another similar low-energy radioisotope, to provide an input signal with a well-known amplitude [5]. Both of these methods, however, have some limitation or drawback: radioisotope spectroscopy cannot be done in-situ, as radioactive sources cannot be placed inside the cryostat due to both spatial limitations and contamination concerns, whilst the PTC method is complicated by phenomena such as the brighter-fatter effect [6] or external noise sources. Cosmic rays can be used to provide an alternative to either of these methods, and this can be done in both laboratory testing or in-situ, where the decreased airmass due to the altitude of typical telescopes increases the muon flux by a factor of about two.

To perform the gain measurement, many dark exposures are taken with the CCD. The choice of integration time is a trade-off between the size of the resulting dataset and the overlapping of tracks in the image, with exposure times in the range of 300-1000s found to be a good compromise. Data was acquired at BNL for a science-grade prototype LSST sensor. The resulting images were then read in and tracks found using DMStack [7], with further data analysis performed in ROOT [8].

\subsection{Track selection}

Once tracks have been found in the dark exposures, a selection is made to exclude spots and worms from the sample whilst keeping muons. Each track is analysed, with several metrics being used to make cuts. For the purpose of gain measurement, tracks are selected on the following basis:

- Straightness - a straight line is fitted to the track, and the linear correlation coefficient $\left(R^{2}\right)$ calculated, with the pixels weighted by intensity. Tracks with a correlation coefficient of $<0.9$ are rejected.

- Length - The diagonal length of the bounding box of the event is required to significantly exceed that of the largest diffusion limited hit, with a typical cut of 100 pixels applied, which also helps filter worms.

- Tracks which cross the edge of the amplifier section are rejected. Because the gain calculation is being performed on an amplifier-by-amplifier basis, tracks which traverse more than one amplifier are discarded for simplicity.

The cuts are optimised for purity over efficiency, with the resulting sample estimated to consist of $>99.5 \%$ muon events.

\subsection{Gain measurement}

The flux in each pixel associated with the muon track is summed, and then divided by the true length of the track in the silicon, accounting for its thickness, to give the energy loss per unit 


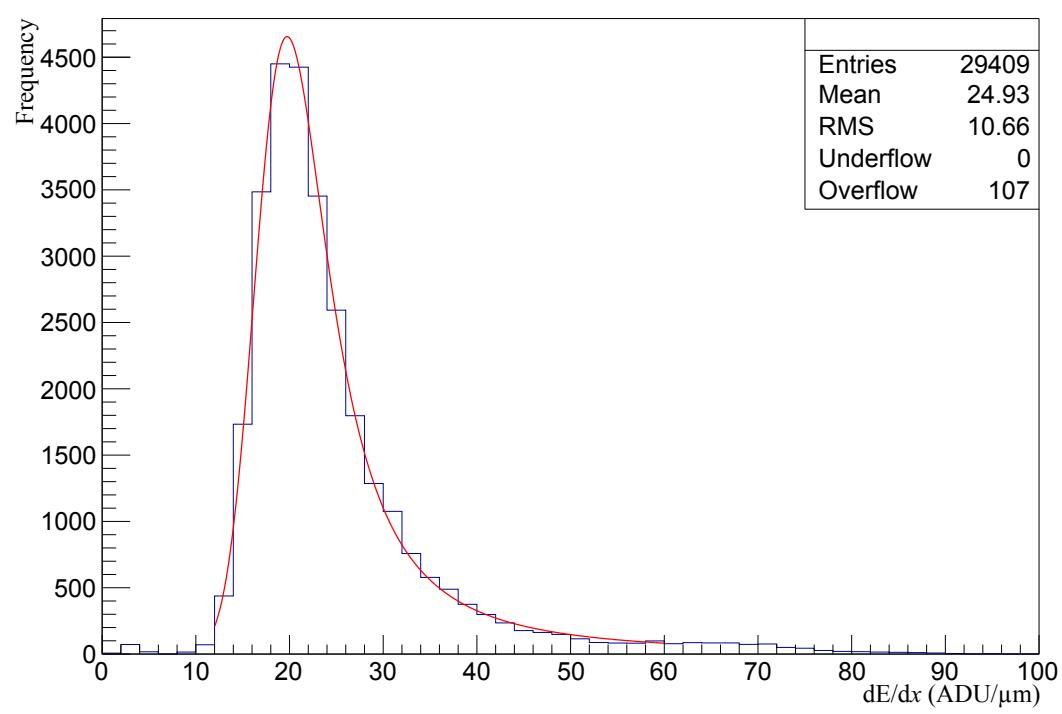

Figure 1. Rate of energy loss, $d E / d x$, for cosmic ray muons across all amplifiers in a $100 \mu \mathrm{m} \mathrm{Si} \mathrm{sensor.} \mathrm{The}$ fit function is a convolution of Landau and Gaussian distributions.

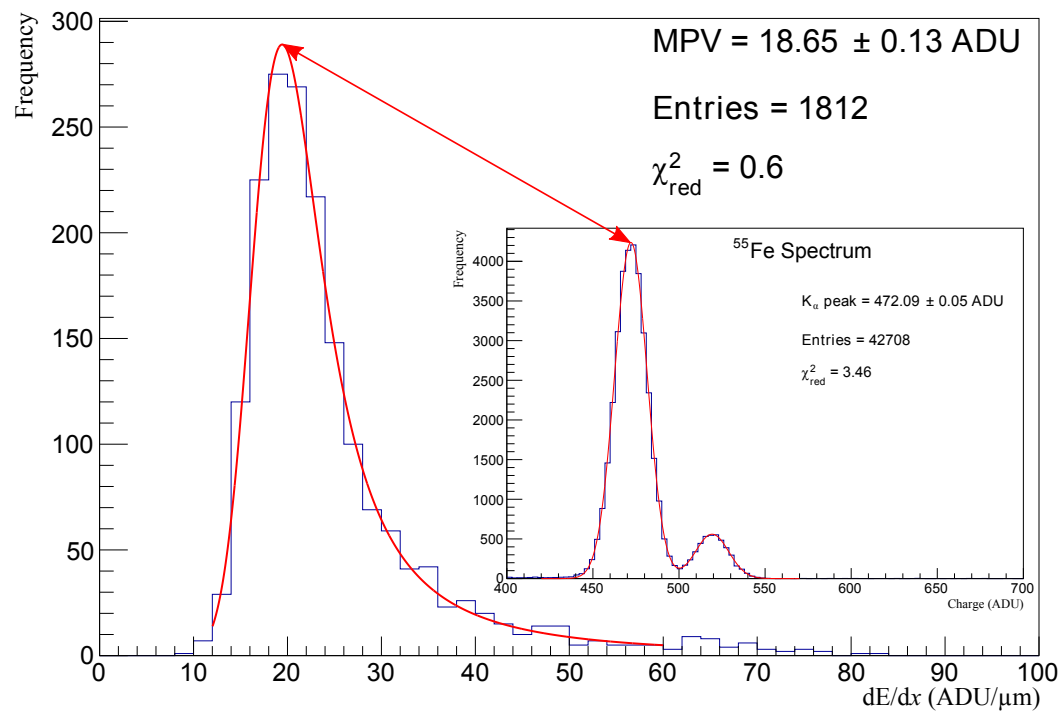

Figure 2. The main histogram shows the muon energy deposition spectrum within a single amplifier section. A fit to the data gives the most-probable energy deposition to better than $1 \%$. The inlay shows the ${ }^{55} \mathrm{Fe}$ energy spectrum in the corresponding section, with the sum of two Gaussians fitted to the peaks.

length, $d E / d x$, of the muon. The most probable value of energy loss (MPV) is calculated from the resulting distribution by fitting a convolution of a Landau and Gaussian distributions. Figure 1 shows the muon energy-loss spectrum for all tracks which pass the cuts for all amplifier sections together before the calibration procedure, and is seen to be well described by the Landau-Gaussian fit. The most probable value from the fit gives the average energy loss for all amplifiers in units of ADU per $\mu \mathrm{m}$.

Producing such a distribution for each amplifier individually allows its relative gain to be calculated. These gains are then plotted against the gain derived from an ${ }^{55} \mathrm{Fe}$ spectrum taken for 


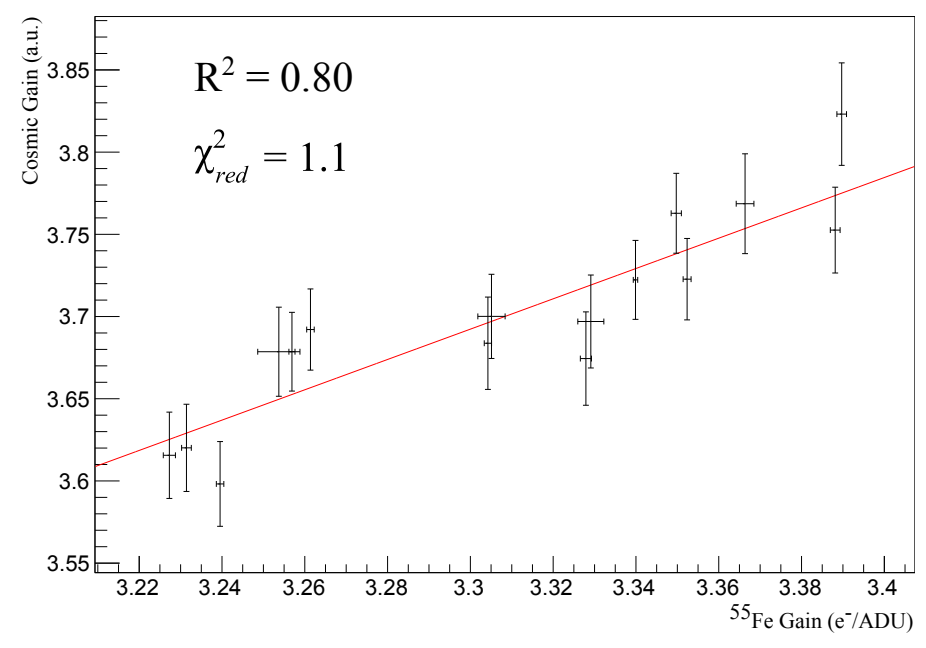

Figure 3. Comparison of the cosmic ray gain measurement to ${ }^{55} \mathrm{Fe}$ measurement technique.

each amplifier, and the correlation calculated. Figure 2 shows the Landau distribution for a single amplifier section, and the corresponding ${ }^{55} \mathrm{Fe}$ spectrum in the inlay. Fifty five hours of integrated darks were used, which yielded $\sim 2,000$ muon tracks per amplifier section after applying the cuts as described. Fitting these distributions allows to determine the MPV to better than $1 \%{ }^{2}$ The corresponding ${ }^{55} \mathrm{Fe}$ spectrum shows the $K_{\alpha}$ and $K_{\beta}$ lines in the ${ }^{55} \mathrm{Fe}$ emission spectrum which have a well-known energy, and a fit to these gives the gain to better than $0.1 \%$, with the dominant source of error being the systematic uncertainty in the pair creation energy in silicon at $-100^{\circ} \mathrm{C}$.

Figure 3 shows the gain calculated from the Landau MPV plotted against the gain derived from the position of the $K_{\alpha}$ peak from the ${ }^{55} \mathrm{Fe}$ measurement. Fitting the data with a straight line shows that the correlation is good, and is consistent with a linear relationship, as expected in the case of good sensor linearity. Errors in the cosmic ray gain measurement are much larger than those for the ${ }^{55} \mathrm{Fe}$ measurement, as the rate at which data can be acquired is a few orders of magnitude lower. However, with larger datasets, the correlation is expected to improve, and the demonstration of linearity confirms that this gain measurement technique is valid.

With respect to converting the cosmic ray gain measurement from arbitrary units to a gain in $\mathrm{e}^{-} /$ADU, careful consideration must be given to the constant of proportionality. Whilst the energy loss spectrum is well described by the Landau distribution, the value for the most probable energy loss is a function of the thickness of the silicon [9], and calculating this is non-trivial, especially as the effective thickness of the sensor is a function of sensor orientation and the cosmic ray angular distribution. The most convenient way is therefore to simply use the linear relationship, and the very precise ${ }^{55} \mathrm{Fe}$ gain calibration, to calculate the MPV for a given sensor configuration, and use this value to tie the MPV in arbitrary units to a value in $\mathrm{e}^{-} /$ADU. This can be done in the lab for a particular sensor geometry, which will then allow the true gain to be calculated in-situ, and will extend to all sensors of that thickness and orientation, for a given altitude. Work is ongoing to make a precise measurement of this value.

\footnotetext{
${ }^{2}$ The error from the fit gives $\Delta_{M P V} / M P V \leq 0.01$.
} 

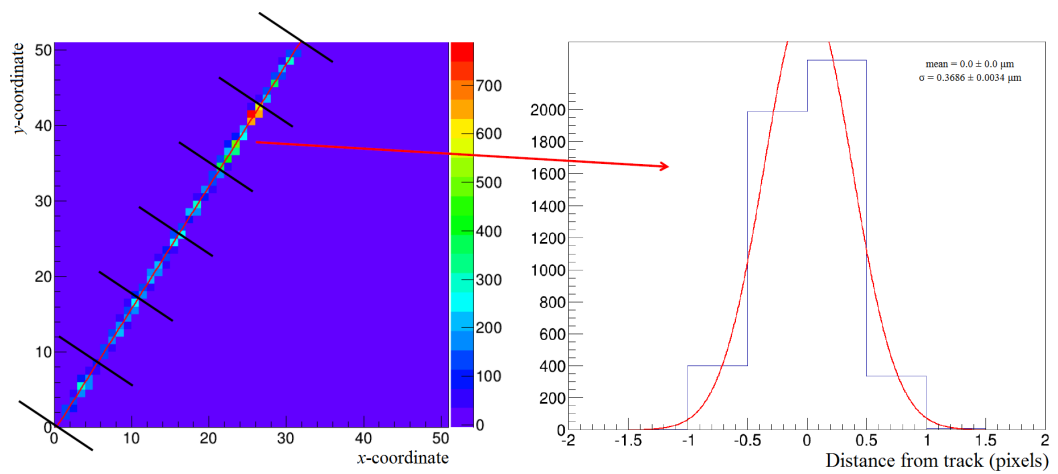

Figure 4. The cosmic ray muon track is divided into sections, with each section's intensity plotted as a function of distance from the track.

\section{PSF and charge diffusion}

Muons which are incident on the sensor at a shallow angle will leave long tracks in the silicon. As the muon passes through the sensor, the drift distance for the deposited charge will vary from zero at the gate-side to the full thickness of the sensor at the back-side. Charges generated furthest away from the gates therefore drift further before collection, and are thus subject to more diffusion, leading to a wider track. By measuring how the width of the track changes along its length, the change in point spread function (PSF) due to the diffusion of charge can be measured.

\subsection{Track selection}

The track selection criteria for making PSF measurement differs slightly to that for gain measurement. Occasionally, as muons traverse the lattice, collisions occur which are so hard that when the atom is ionised, the freed electron is given enough energy to leave an ionisation trail itself. These electrons are referred to as $\delta$-rays, and they contribute to the long tail of the Landau distribution. Whilst it is important to sum the contribution from these $\delta$-rays when measuring gain, one must exclude tracks containing long $\delta$-rays from the selection when measuring PSF, as they will necessarily be depositing charge far away from the track centroid, leading to a wider track. Therefore, when making the track selection for PSF measurement, tracks are selected as before, to be straight and long in order to eliminate worms and spots whilst including muons, but they must also have no measurable $\delta$-rays protruding from the track. $\delta$-rays are excluded by calculating a special discriminatory parameter, where pixel intensities are weighted exponentially by their distance from the track, causing the value for tracks with $\delta$-rays to quickly diverge. Finally, tracks are required not cross the edge of the sensor, as otherwise the exit depth of the muon is unknown. ${ }^{3}$

\subsection{PSF measurement}

Having made the track selection, the PSF as a function of thickness is measured as follows. Each track is fitted with a straight line, and is then divided into several sections, each of which will

\footnotetext{
${ }^{3}$ The muons are assumed to be fully penetrating, so if a track does not cross the sensor's edge then one end must correspond to zero depth, and the other to full depth. If, however, the track crosses the edge of the sensor, its depth in the silicon at the point of exit is unknown.
} 


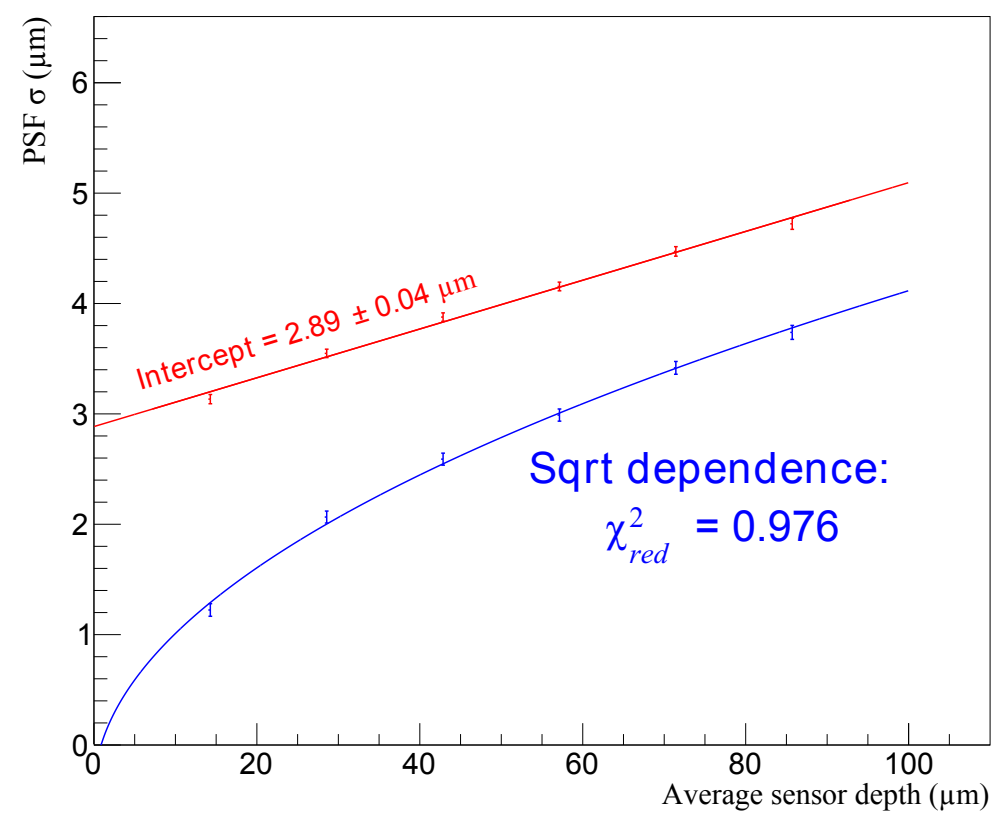

Figure 5. PSF as a function of depth in the sensor. The red points show the total PSF at that depth, whilst the blue points mark the contribution from charge diffusion.

correspond to some average depth in the silicon. For this track section, the intensity is plotted as a function of distance from the fit to the track, producing an approximately normal distribution, as shown in figure 4 . This distribution is then fitted with a Gaussian, the width of which is plotted as a function of the depth in the silicon for each track section.

At this stage, it is not known in which direction the muon was travelling with respect to the sensor's surface, so there is ambiguity in the which end corresponds to zero depth and which to full thickness. An arbitrary initial direction is assumed, and the PSF as a function of depth is fitted with a straight line. The gradient of this line is then used to orientate the track with respect to the sensor surface, with the 'thick-end' being assumed to correspond to the longest drift. If the error on the gradient of this line is larger than the gradient itself, the track's orientation is considered ambiguous, and it is discarded from the sample. Once all tracks are aligned, the PSFs at a given depth are averaged together, and the PSF is plotted as a function of energy deposition depth, as shown in figure 5.

Assuming the volume of the ionisation trail to be infinitesimal, the two components of the total PSF, $\sigma$, are the charge diffusion, $\sigma_{d i f f}$ and the intrinsic resolution of the pixel detector, $\sigma_{0}$, where

$$
\sigma=\sqrt{\sigma_{0}^{2}+\sigma_{d i f f}^{2}}
$$

and the intrinsic resolution is simply [10]

$$
\sigma_{0}=\frac{\text { pixel pitch }}{\sqrt{12}}
$$

The charge diffuses continually as it drifts, with the diffusive PSF as a function of drift distance given by $[10,11]$

$$
\sigma_{\text {diff }}(\Delta z)=\sqrt{2 \frac{k T}{e} \frac{D \Delta z}{V}}
$$



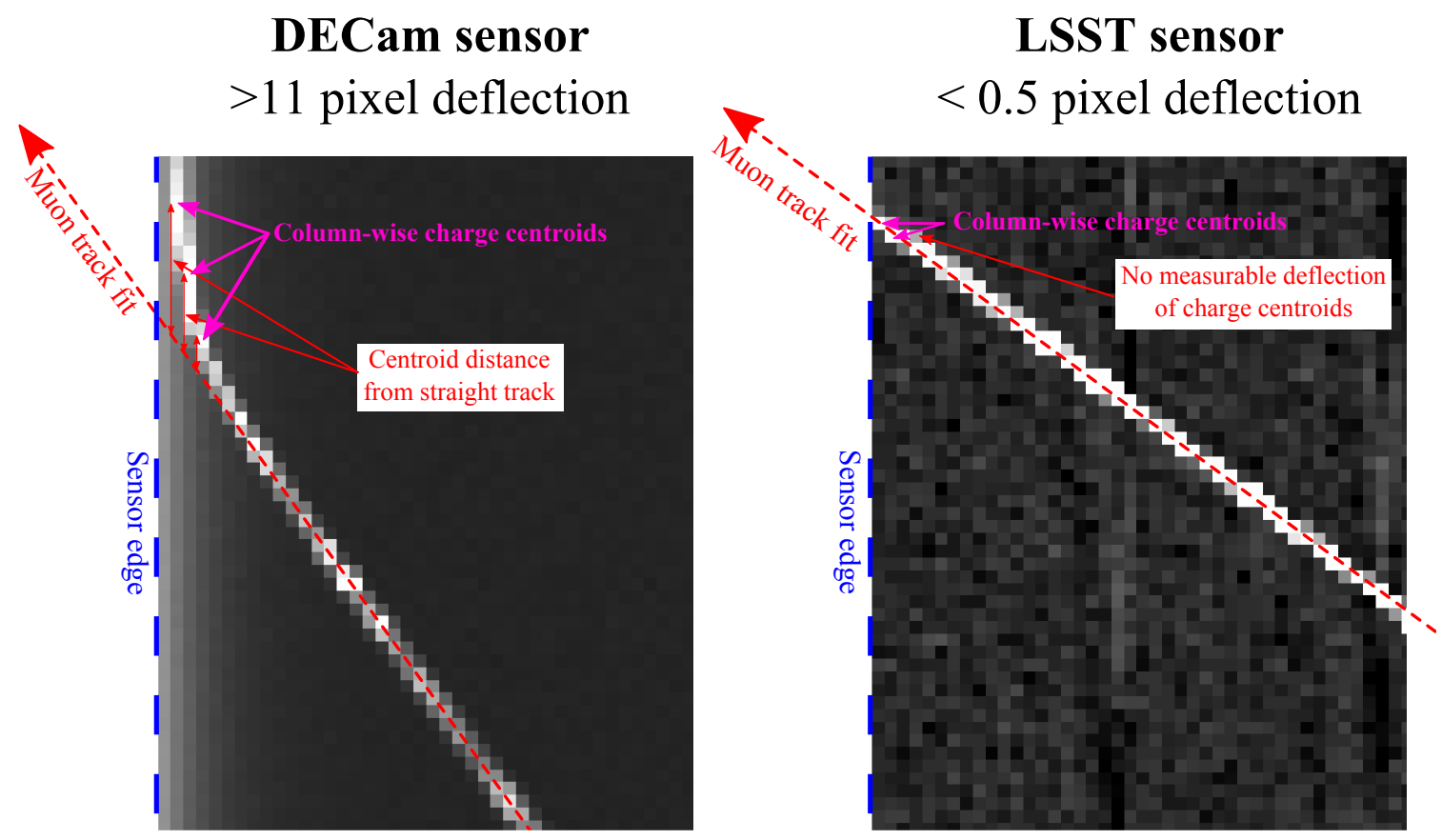

Figure 6. Track "bending" at sensor edges in DECam and LSST sensors.

where $D$ is the detector thickness, $V$ is the applied voltage and $\Delta z$ is the depth in the sensor at which charge is generated, i.e. the drift distance.

Fitting a straight line to the red points in figure 5 and extrapolating shows that $\sigma_{0}$ is equal to $2.89 \mu \mathrm{m}$, in good agreement with (3.2), as the pitch of the LSST sensors is $10 \mu \mathrm{m}$, corresponding to $\sigma_{0}=10 \mu \mathrm{m} / \sqrt{12}=2.89 \mu \mathrm{m}$. Subtracting $\sigma_{0}$ in quadrature allows the depth-dependent drift contribution to be separated, as shown by the blue points in figure 5 , where a $\sqrt{\Delta z}$ function has been fitted, showing good agreement with theory. The overall trend in PSF with respect to depth is that of an approximately linear increase with respect to drift distance, at a rate of $0.02 \mu \mathrm{m}$ per $\mu \mathrm{m}$ of drift.

\section{Edge straightness}

Static electric fields are typically found near the edges of CCD sensors due to the guard-rings around their perimeters. These fields can cause charges to move laterally, sometimes by many pixels, causing the tracks of cosmic rays to appear visibly bent. Whilst examination of the shape of cosmic ray tracks is a relatively insensitive probe compared to precision measurements like ${ }^{55} \mathrm{Fe}$ cluster shape analysis [12], examination of cosmic ray tracks which cross the edges of sensors can give an at-a-glance indication of whether large lateral electrics fields have been induced near the sensor's edge.

The sensors used in the Dark Energy Camera (DECam) [13] suffer from significant 'baked-in' lateral electric fields, i.e. they are a product of the fabrication process and are not dynamical in nature. The left pane of figure 6 shows a cosmic ray track crossing the edge of a DECam sensor in a public DECam dark exposure. The columns at the edge of the sensor are seen to 'glow', which 


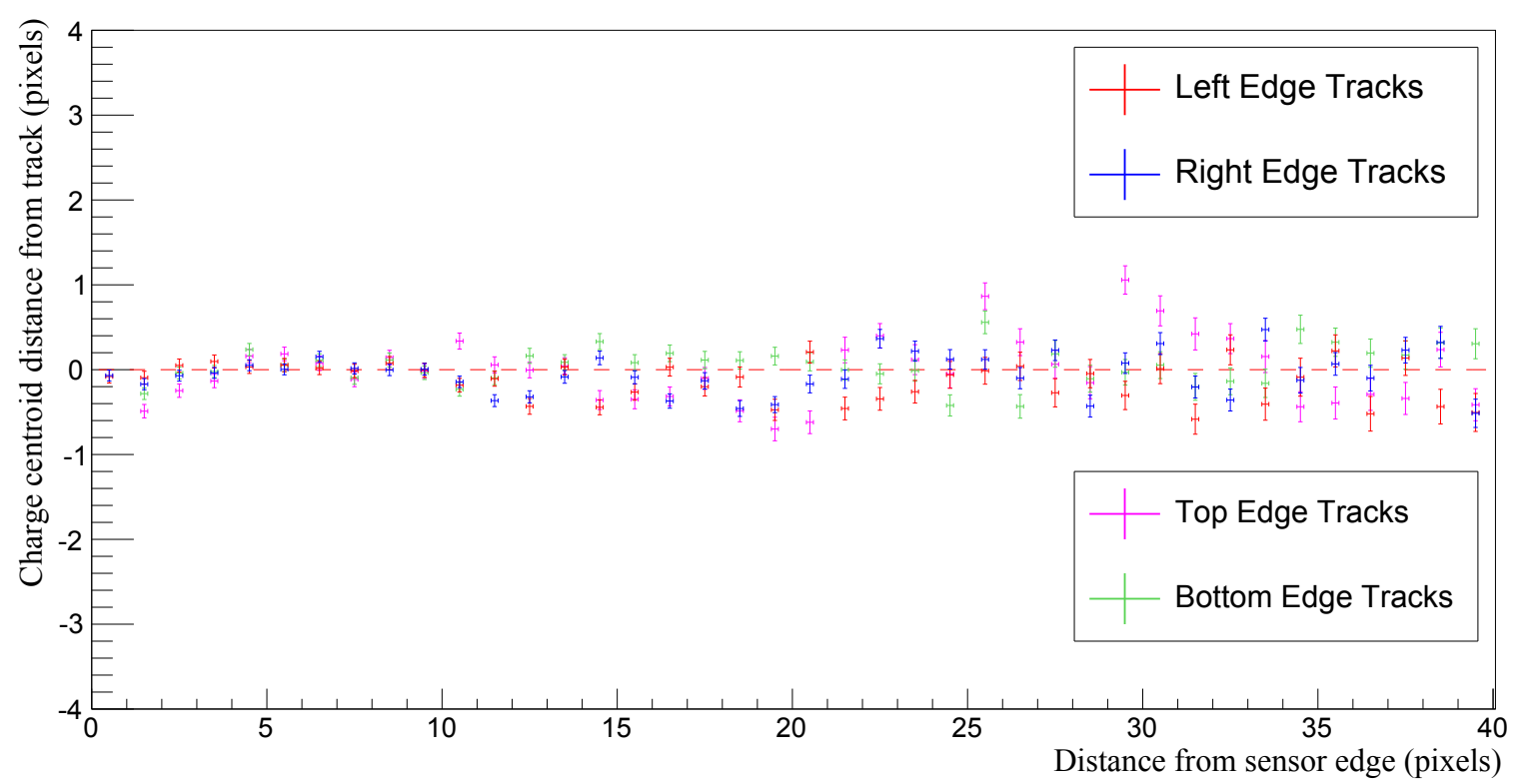

Figure 7. Deviation of the charge centroid position with respect to distance from edge of the sensor, for muon tracks crossing the edge of a prototype LSST sensor.

is attributed to the fields from the guard-rings and the properties of the front-gate electrodes [14]. These lateral electric fields can be seen to visibly 'bend' the track, deflecting the charge away from the edge of the sensor. The right pane of figure 6 shows a similar edge-crossing track in a prototype LSST sensor, where no visible bending is seen.

In order to make a quantitative measurement of track bending, the charge centroid is calculated for each column, and its distance to the track's fit is plotted with respect to the column's distance from the sensor's edge, with the same analysis performed row-wise for tracks crossing the top and bottom of the sensor. Figure 7 shows the measurement of these deviations for a prototype LSST sensor. The error bars increase in size as the distance from the edge increases, due to the lower number of tracks which extend this far. Whilst this only provides a crude assessment of the distortions at the sensor's edges, the measurements show that there is no visible bending of tracks, which is in contrast with column centroid deviations of more than 10 pixels in DECam, ${ }^{4}$ showing that the prototype LSST sensors have a considerably smaller edge effect.

\section{Summary}

The properties of cosmic ray muons make them a powerful tool for use in the characterisation of astronomical CCD sensors. With sufficient data, these tracks can be used to measure the gain of the sensors despite the stochastic nature of the energy deposition, and this can be done "for free" on the mountain during the day. The small area over which the muons deposit their energy, combined with the straightness of the tracks, provides a tool to directly measure the diffusion of charge as it crosses the sensor. And, though other techniques are considerably more sensitive to

\footnotetext{
${ }^{4}$ The 10 pixel deviation observed in DECam sensors would be equivalent to a 15 pixel shift in LSST sensors due to the smaller pixel pitch in LSST.
} 
these effects, the straightness of the tracks at the sensor's edges provides a quick guide to potential edge-distortions arising from lateral electric fields due to guard-rings.

\section{Acknowledgments}

The authors would like to thank Paul O'Connor and Ivan Kotov for their help in the collection of the dark image data, Juan Estrada for his ideas and discussion regarding the measurement of PSF and charge diffusion using cosmic rays, and Robert Lupton, Paul Price, and the DMStack Team for their help in getting to grips with the DMStack image processing framework.

\section{References}

[1] D. Groom, Cosmic rays and other nonsense in astronomical CCD imagers, Exper. Astron. 14 (2002) 45 .

[2] Particle Data Group collaboration, J. Beringer et al., Review of particle physics, Phys. Rev. D 86 (2012) 323.

[3] S.M. Kahn et al., Design and development of the 3.2 gigapixel camera for the large synoptic survey telescope, (2010).

[4] J.R. Janesick, Photon Transfer, SPIE-Intl Soc Optical Eng, (2007).

[5] J. Janesick, Scientific Charge-coupled Devices, Press Monographs, Society of Photo Optical (2001).

[6] P. Antilogus, P. Astier, P. Doherty, A. Guyonnet and N. Regnault, The brighter-fatter effect and pixel correlations in CCD sensors, 2014 JINST 9 C03048.

[7] T. Axelrod, J. Kantor, R. H. Lupton and F. Pierfederici, An open source application framework for astronomical imaging pipelines, Proc. SPIE 7740 (2010) 774015.

[8] R. Brun and F. Rademakers, ROOT: An object oriented data analysis framework, Nucl. Instrum. Meth. A 389 (1997) 81.

[9] H. Bichsel, Straggling in Thin Silicon Detectors, Rev. Mod. Phys. 60 (1988) 663 [inSPIRE].

[10] H. Spieler, Semiconductor detector systems, vol. 12, Oxford University Press, Oxford U.K. (2005).

[11] P. Koppenburg, A simulation of charge deposition and collection in silicon microstrip detectors, LHCb VeLo 42 (2001) 2001.

[12] A. Nomerotski, New characterization techniques for LSST sensors, in Precision Astronomy with Fully Depleted CCDs, 4-5 December 2014, Brookhaven National Laboratory, U.S.A.

[13] R.A. Shaw (ed.), NOAO Data Handbook, Version 2.1, National Optical Astronomy Observatory, Tucson U.S.A. (2015).

[14] A.A. Plazas, G.M. Bernstein and E.S. Sheldon, On-sky measurements of the transverse electric fields' effects in the Dark Energy Camera CCDs, Publ. Astron. Soc. Pac. 126 (2014) 750. 